\title{
Graded Morita theory over a G-graded G-acted algebra
}

\author{
Virgilius-Aurelian Minuță \\ Babeș-Bolyai University of Cluj-Napoca \\ Faculty of Mathematics and Computer Science \\ Department of Mathematics \\ email: minuta.aurelian@math.ubbcluj.ro
}

\begin{abstract}
We develop a group graded Morita theory over a G-graded $\mathrm{G}$-acted algebra, where $\mathrm{G}$ is a finite group.
\end{abstract}

\section{Introduction}

Let $\mathrm{G}$ be a finite group. In this article we develop a group graded Morita theory over a G-graded G-acted algebra, which is motivated by the problem to give a group graded Morita equivalences version of relations between character triples (see [11]) as in [9]. We will follow, in the development of graded Morita theory over a G-graded G-acted algebra, the treatment of Morita theory given by C. Faith in 1973 in [5]. Significant in this article is the already developed graded Morita theory. Graded Morita theory started in 1980 when R. Gordon and E. $\mathrm{L}$. Green have characterized graded equivalences in the case of $\mathrm{G}=\mathbb{Z}$, in [6]. Furthermore, in 1988 it was observed to work for arbitrary groups G by C. Menini and C. Năstăsescu, in [10]. We will make use of their results under the form given by A. del Río in 1991 in [4] and we will also use the graded Morita theory developed by P. Boisen in 1994 in [2].

2010 Mathematics Subject Classification: 16W50, 16D20, 16D90, 16S35

Key words and phrases: crossed product, group graded Morita equivalence, centralizer subalgebra, graded rings and modules 
This paper is organized as follows: In Section §2, we introduce the general notations. In Section §3, we recall from [9] the definition of a G-graded Gacted algebra, we fix one to which we will further refer to by $\mathscr{C}$, we recall the definition of a G-graded algebra over said $\mathscr{C}$, and we recall the definition of a graded bimodule over $\mathscr{C}$. Moreover, we will give some new examples, useful for this article, for each notion. In Section $\S 4$, we construct the notion of a G-graded Morita context over $\mathscr{C}$ and we will give an appropriate example. In Section $\S 5$, we introduce the notions of graded functors over $\mathscr{C}$ and of graded Morita equivalences over $\mathscr{C}$ and finally we state and prove two Morita-type theorems using the said notions.

\section{Notations and preliminaries}

Throughout this article, we will consider a finite group G. We shall denote its identity by 1 .

All rings in this paper are associative with identity $1 \neq 0$ and all modules are unital and finitely generated. We consider $\mathcal{O}$ to be a commutative ring.

Let $A$ be a ring. We denote by $A$-Mod the category of all left $A$-modules. We shall usually write actions on the left, so in particular, by module we will usually mean a left module, unless otherwise stated. The notation ${ }_{A} M$ (respectively, ${ }_{A} M_{A^{\prime}}$ ) will be used to emphasize that $M$ is a left $A$-module (respectively, an $\left(A, A^{\prime}\right)$-bimodule).

Let $A=\bigoplus_{g \in G} A_{g}$ be a G-graded $\mathcal{O}$-algebra. We denote by $A$-Gr the category of all G-graded left A-modules. The forgetful functor from A-Gr to A-Mod will be denoted by $\mathrm{U}$. For $M=\bigoplus_{\mathrm{g} \in \mathrm{G}} \mathrm{M}_{\mathrm{g}} \in \mathrm{A}$-Gr and $\mathrm{g} \in \mathrm{G}$, the $\mathrm{g}$-suspension of $M$ is defined to be the G-graded A-module $M(g)=\oplus_{h \in G} M(g)_{h}$, where $\mathrm{M}(\mathrm{g})_{\mathrm{h}}=\mathrm{M}_{\mathrm{gh}}$. For any $\mathrm{g} \in \mathrm{G}, \mathrm{T}_{\mathrm{g}}^{\mathrm{A}}: \mathrm{A}-\mathrm{Gr} \rightarrow \mathrm{A}$-Gr will denote (as in [4]) the $\mathrm{g}$-suspension functor, i.e. $T_{\mathrm{g}}^{A}(M)=M(\mathrm{~g})$ for all $\mathrm{g} \in \mathrm{G}$. The stabilizer of $M$ in $\mathrm{G}$ is, by definition $[7, \S 2.2 .1]$, the subgroup

$$
\mathrm{G}_{\mathrm{M}}=\{\mathrm{g} \in \mathrm{G} \mid \mathrm{M} \simeq \mathrm{M}(\mathrm{g}) \text { as } \mathrm{G} \text {-graded left } \mathrm{A} \text {-modules }\} .
$$

Let $M, N \in A$-Gr. We denote by $\operatorname{Hom}_{A}(M, N)$, the additive group of all $A$ linear homomorphisms from $M$ to $N$. Because $G$ is finite, E. C. Dade showed in [3, Corollary 3.10] that $\operatorname{Hom}_{\mathcal{A}}(M, N)$ is G-graded. More precisely, if $g \in G$, the component of degree $\mathrm{g}$ (furthermore called the $\mathrm{g}$-component) is defined as in $[7,1.2]$ :

$$
\operatorname{Hom}_{\mathcal{A}}(M, N)_{g}:=\left\{f \in \operatorname{Hom}_{\mathcal{A}}(M, N) \mid f\left(M_{x}\right) \subseteq N_{x g} \text {, for all } x \in G\right\} .
$$


We denote by idx the identity map defined on a set $X$.

\section{Graded bimodules over a G-graded G-acted algebra}

We consider the notations given in Section $\S 2$. We recall the definition of a G-graded G-acted algebra and an example of a G-graded G-acted algebra as in [9]:

Definition 1 An algebra $\mathscr{C}$ is a G-graded G-acted algebra if

1. $\mathscr{C}$ is G-graded, i.e. $\mathscr{C}=\oplus_{\mathrm{g} \in \mathrm{G}} \mathscr{C}_{\mathrm{g}}$;

2. $\mathrm{G}$ acts on $\mathscr{C}$ (always on the left in this article);

3. $\forall \mathrm{h} \in \mathrm{G}, \forall \mathrm{c} \in \mathscr{C}_{\mathrm{h}}$ we have that ${ }^{{ }^{\mathrm{c}}} \in \mathscr{C}_{{ }_{\mathrm{gh}}}$ for all $\mathrm{g} \in \mathrm{G}$.

We denote the identity component (the 1-component) of $\mathscr{C}$ by $\mathscr{Z}:=\mathscr{C}_{1}$, which is a $\mathrm{G}$-acted algebra.

Let $A=\bigoplus_{g \in G} A_{g}$ be a strongly G-graded $\mathcal{O}$-algebra with identity component $B:=A_{1}$. For the sake of simplicity, we assume that $A$ is a crossed product (the generalization is not difficult, see for instance [7, §1.4.B.]). This means that we can choose invertible homogeneous elements $u_{g}$ in the component $A_{g}$.

Example 1 By Miyashita's theorem [7, p.22], we know that the centralizer $\mathrm{C}_{\mathrm{A}}(\mathrm{B})$ is a $\mathrm{G}$-graded $\mathrm{G}$-acted $\mathcal{O}$-algebra: for all $\mathrm{h} \in \mathrm{G}$ we have that

$$
\mathrm{C}_{\mathrm{A}}(\mathrm{B})_{\mathrm{h}}=\left\{\mathrm{a} \in \mathrm{A}_{\mathrm{h}} \mid \mathrm{ab}=\mathrm{ba}, \forall \mathrm{b} \in \mathrm{B}\right\},
$$

and the action is given by ${ }^{\mathrm{g}} \mathrm{c}=\mathrm{u}_{\mathrm{g}} \mathrm{cu}_{\mathrm{g}}^{-1}, \forall \mathrm{g} \in \mathrm{G}, \forall \mathrm{c} \in \mathrm{C}_{\mathrm{A}}(\mathrm{B})$. Note that this definition does not depend on the choice of the elements $\mathfrak{u}_{\mathrm{g}}$ and that $\mathrm{C}_{\mathrm{A}}(\mathrm{B})_{1}=\mathrm{Z}(\mathrm{B})$ (the center of $\mathrm{B}$ ).

We recall the definition of a $G$-graded $\mathcal{O}$-algebra over a $\mathrm{G}$-graded G-acted algebra $\mathscr{C}$ as in [9]:

Definition 2 Let $\mathscr{C}$ be a $\mathrm{G}$-graded $\mathrm{G}$-acted $\mathcal{O}$-algebra. We say that $\mathrm{A}$ is a $\mathrm{G}$ graded $\mathcal{O}$-algebra over $\mathscr{C}$ if there is a $\mathrm{G}$-graded $\mathrm{G}$-acted algebra homomorphism

$$
\zeta: \mathscr{C} \rightarrow \mathrm{C}_{\mathrm{A}}(\mathrm{B}),
$$

i.e. for any $\mathrm{h} \in \mathrm{G}$ and $\mathrm{c} \in \mathscr{C}_{\mathrm{h}}$, we have $\zeta(\mathrm{c}) \in \mathrm{C}_{\mathrm{A}}(\mathrm{B})_{\mathrm{h}}$, and for every $\mathrm{g} \in \mathrm{G}$, we have $\zeta\left({ }^{\mathrm{g}} \mathrm{c}\right)={ }^{\mathrm{g}} \zeta(\mathrm{c})$. 
An important example of a $\mathrm{G}$-graded $\mathcal{O}$-algebra over a G-graded G-acted algebra, is given by the following lemma:

Lemma 1 Let $\mathrm{P}$ be a $\mathrm{G}$-graded $\mathrm{A}$-module. Assume that $\mathrm{P}$ is $\mathrm{G}$-invariant. Let $\mathrm{A}^{\prime}=\operatorname{End}_{\mathcal{A}}(\mathrm{P})^{o p}$ be the set of all $\mathrm{A}$-linear endomorphisms of $\mathrm{P}$. Then $\mathrm{A}^{\prime}$ is a $\mathrm{G}$-graded $\mathcal{O}$-algebra over $\mathrm{C}_{\mathrm{A}}(\mathrm{B})$.

Proof. By [3, Theorem 2.8], we have that there exists some $\mathrm{U} \in \mathrm{B}-\bmod$ such that $\mathrm{P}$ and $\mathrm{A} \otimes_{\mathrm{B}} \mathrm{U}$ are isomorphic as G-graded left $A$-modules, henceforth for simplicity we will identify $\mathrm{P}$ as $\mathrm{A} \otimes_{\mathrm{B}} \mathrm{U}$.

Because $G$ is finite, E. C. Dade proved in [3, Corollary 3.10 and $\S 4]$ that $A^{\prime}=\operatorname{End}_{A}(P)^{\text {op }}$ is a $\mathrm{G}$-graded $\mathcal{O}$-algebra. Moreover, by $[3, \S 4]$ we have that $\mathrm{P}$ is actually a $\mathrm{G}$-graded $\left(A, A^{\prime}\right)$-bimodule.

Now, the assumption that $P$ is G-invariant, according to [3, Corollary 5.14] and $[7, \S 2.2 .1]$, implies that $A^{\prime}=\operatorname{End}_{A}(P)^{\text {op }}$ is a crossed product and that $P$ is isomorphic to its $\mathrm{g}$-suspension, $\mathrm{P}(\mathrm{g})$, for all $\mathrm{g} \in \mathrm{G}$. Henceforth, we can choose invertible homogeneous elements $u_{g}^{\prime}$ in the component $A_{g}^{\prime}$, for all $g \in G$ such that

$$
\mathrm{u}_{\mathrm{g}}^{\prime}: \mathrm{P} \rightarrow \mathrm{P}(\mathrm{g}) \text {. }
$$

By taking the truncation functor $(-)_{1}$ (more details are given in [3]) we obtain the isomorphism:

$$
\left(u_{g}^{\prime}\right)_{1}: P_{1} \rightarrow(P(g))_{1},
$$

where $P_{1}=B \otimes_{B} U \simeq U$ and $(P(g))_{1}=A_{g} \otimes_{B} U=u_{g} B \otimes_{B} U$. We fix arbitrary $a \in A$ and $u \in U$. We have:

$$
u_{g}^{\prime}\left(a \otimes_{B} u\right)=a u_{g}^{\prime}\left(1_{A} \otimes_{B} u\right),
$$

but $1_{\mathrm{A}} \otimes_{\mathrm{B}} u \in \mathrm{P}_{1}$, henceforth:

$$
u_{g}^{\prime}\left(a \otimes_{B} u\right)=a\left(u_{g}^{\prime}\right)_{1}\left(1_{A} \otimes_{B} u\right),
$$

but there exists an unique $b \in B$ such that $\left(u_{g}^{\prime}\right)_{1}\left(1_{A} \otimes_{B} \mathfrak{u}\right)=u_{g} b \otimes_{B} u=$ $u_{\mathrm{g}} \otimes_{\mathrm{B}}$ bu. Therefore, by defining $\varphi_{\mathrm{g}}(\mathrm{u}):=\mathrm{bu}$, we obtain a map $\varphi_{\mathrm{g}}: \mathrm{U} \rightarrow \mathrm{U}$, which is clearly well-defined. Moreover, we have:

$$
u_{g}^{\prime}\left(a \otimes_{B} u\right)=a u_{g} \otimes_{B} \varphi_{g}(u) \text {, for all } a \in A \text { and } u \in U \text {. }
$$

It is straightforward to prove that $\varphi_{\mathrm{g}}: \mathrm{U} \rightarrow \mathrm{U}$ admits an inverse and that

$$
u_{g}^{\prime-1}\left(a \otimes_{B} u\right)=a u_{g}^{-1} \otimes_{B} \varphi_{g}^{-1}(u), \text { for all } a \in A \text { and } u \in U \text {. }
$$


We consider the G-graded algebra homomorphism from [8, Lemma 3.2.]:

$$
\theta: C_{A}(B) \rightarrow A^{\prime}=\operatorname{End}_{A}(P)^{o p}, \quad \theta(c)(a \otimes u)=a c \otimes u,
$$

where $c \in C_{A}(B), a \in A$, and $u \in U$. First, we will prove that the image of $\theta$ is a subset of $C_{A^{\prime}}\left(B^{\prime}\right)$. Indeed, consider $b^{\prime} \in B^{\prime}$ and $c \in C_{A}(B)$. We want:

$$
\theta(c) \circ b^{\prime}=b^{\prime} \circ \theta(c) .
$$

Consider $a \otimes u \in A \otimes U=P$. We have:

$$
\left(\theta(c) \circ b^{\prime}\right)(a \otimes u)=\theta(c)\left(b^{\prime}(a \otimes u)\right)=a \theta(c)\left(b^{\prime}\left(1_{A} \otimes u\right)\right),
$$

because $b^{\prime}$ and $\theta(c)$ are $A$-linear. We fix $b^{\prime}\left(1_{A} \otimes u\right)=a_{0} \otimes u_{0} \in A \otimes_{B} \mathrm{U}$, but because $b^{\prime} \in B^{\prime}=A_{1}^{\prime}=\operatorname{End}_{A}(P)_{1}^{\text {op }}$ we know that $b^{\prime}$ preserves the grading, so $1_{\mathrm{A}} \in A_{1}$ implies that $a_{\mathrm{o}} \in \mathrm{B}$. Hence:

$$
\left(\theta(c) \circ b^{\prime}\right)(a \otimes u)=a \theta(c)\left(a_{0} \otimes u_{0}\right)=a a_{0} c \otimes u_{0}=a c a_{0} \otimes u_{0} .
$$

Following, we have that:

$$
\begin{aligned}
\left(b^{\prime} \circ \theta(c)\right)(a \otimes u) & =\left(b^{\prime}(\theta(c)(a \otimes u))=b^{\prime}(a c \otimes u)\right. \\
& =a c b^{\prime}\left(1_{A} \otimes u\right)=a c a_{0} \otimes u_{0} .
\end{aligned}
$$

Henceforth, the image of $\theta$ is a subset of $C_{A^{\prime}}\left(B^{\prime}\right)$. Second, we prove that $\theta$ is G-acted, in the sense that:

$$
\theta\left({ }^{g} \mathbf{c}\right)={ }^{g}(\theta(\mathbf{c})), \text { for all } \mathrm{g} \in \mathrm{G} .
$$

Indeed, we fix $g \in G$, and $a \otimes u \in A \otimes_{B} U=P$. We have:

$$
\theta\left({ }^{g} \mathfrak{c}\right)(\mathfrak{a} \otimes \mathfrak{u})=\theta\left(\mathfrak{u}_{g} c u_{g}^{-1}\right)(\mathfrak{a} \otimes \mathfrak{u})=\mathfrak{a u}_{g} \mathfrak{c u}_{g}^{-1} \otimes \mathfrak{u}
$$

and

$$
\begin{aligned}
{ }^{g}(\theta(c))(a \otimes u) & =\left(u_{g}^{\prime} \cdot \theta(c) \cdot u_{g}^{\prime-1}\right)(a \otimes u)=\left(u_{g}^{\prime-1} \circ \theta(c) \circ u_{g}^{\prime}\right)(a \otimes u) \\
& =u_{g}^{\prime-1}\left(\theta(c)\left(u_{g}^{\prime}(a \otimes u)\right)=u_{g}^{\prime-1}\left(\theta(c)\left(a u_{g} \otimes \varphi_{g}(u)\right)\right)\right. \\
& =u_{g}^{\prime-1}\left(a u_{g} c \otimes \varphi_{g}(u)\right)=a u_{g} c u_{g}^{-1} \otimes \varphi_{g}^{-1}\left(\varphi_{g}(u)\right) \\
& =a u_{g} c u_{g}^{-1} \otimes u .
\end{aligned}
$$

Finally, by taking $\zeta^{\prime}: C_{A}(B) \rightarrow C_{A^{\prime}}\left(B^{\prime}\right)$ to be the corestriction of $\theta$ to $C_{A^{\prime}}\left(B^{\prime}\right)$, we obtain that $A^{\prime}$ is G-graded $\mathcal{O}$-algebra over $C_{A}(B)$, via the Ggraded G-acted homomorphism $\zeta^{\prime}$. 
Let $A^{\prime}=\bigoplus_{g \in G} A_{g}^{\prime}$ be another strongly G-graded $\mathcal{O}$-algebra with the identity component $B^{\prime}:=A_{1}^{\prime}$. Again, we will consider that also $A^{\prime}$ is a crossed product, hence we will choose invertible homogeneous elements $u_{g}^{\prime}$ in the component $A_{g}^{\prime}$, for all $g \in G$.

Now, we assume that $A$ and $A^{\prime}$ are both strongly G-graded $\mathcal{O}$-algebras over a G-graded G-acted algebra $\mathscr{C}$, each endowed with G-graded G-acted algebra homomorphism $\zeta: \mathscr{C} \rightarrow \mathrm{C}_{\mathrm{A}}(\mathrm{B})$ and $\zeta^{\prime}: \mathscr{C} \rightarrow \mathrm{C}_{\mathrm{A}^{\prime}}\left(\mathrm{B}^{\prime}\right)$ respectively.

We recall the definition of a G-graded bimodule over $\mathscr{C}$ as in [9]:

Definition 3 We say that $\tilde{M}$ is a $\mathrm{G}$-graded $\left(A, A^{\prime}\right)$-bimodule over $\mathscr{C}$ if:

1. $\tilde{M}$ is an $\left(A, A^{\prime}\right)$-bimodule;

2. $\tilde{M}$ has a decomposition $\tilde{M}=\bigoplus_{g \in G} \tilde{M}_{g}$ such that $A_{g} \tilde{M}_{x} A_{h}^{\prime} \subseteq \tilde{M}_{g x h}$, for all $\mathrm{g}, \mathrm{x}, \mathrm{h} \in \mathrm{G}$;

3. $\tilde{\mathrm{m}}_{\mathrm{g}} \cdot \mathrm{c}={ }^{\mathrm{g}} \mathrm{c} \cdot \tilde{\mathrm{m}}_{\mathrm{g}}$, for all $\mathrm{c} \in \mathscr{C}, \tilde{\mathrm{m}}_{\mathrm{g}} \in \tilde{\mathrm{M}}_{\mathrm{g}}, \mathrm{g} \in \mathrm{G}$, where $\mathrm{c} \cdot \tilde{\mathrm{m}}=\zeta(\mathrm{c}) \cdot \tilde{\mathrm{m}}$ and $\tilde{\mathrm{m}} \cdot \mathrm{c}=\tilde{\mathrm{m}} \cdot \zeta^{\prime}(\mathrm{c})$, for all $\mathrm{c} \in \mathscr{C}, \tilde{\mathrm{m}} \in \tilde{M}$.

Remark 1 Condition 3. of Definition 3 can be rewritten (see [9] for the proof) as follows:

3'. $\mathrm{m} \cdot \mathrm{c}=\mathrm{c} \cdot \mathrm{m}$, for all $\mathrm{c} \in \mathscr{C}, \mathrm{m} \in \tilde{\mathrm{M}}_{1}$.

An example of a G-graded bimodule over a G-graded G-acted algebra is given by the following proposition:

Proposition 1 Let $\mathscr{C}$ be a G-graded G-acted algebra and A a strongly Ggraded $\mathcal{O}$-algebra over $\mathscr{C}$. Let $\mathrm{P}$ be a $\mathrm{G}$-invariant $\mathrm{G}$-graded $\mathrm{A}$-module. Let $A^{\prime}=\operatorname{End}_{A}(P)^{o p}$. Then the following statements hold:

1. $\mathrm{A}^{\prime}$ is a $\mathrm{G}$-graded $\mathcal{O}$-algebra over $\mathscr{C}$;

2. $\mathrm{P}$ is a $\mathrm{G}$-graded $\left(\mathrm{A}, \mathrm{A}^{\prime}\right)$-bimodule over $\mathscr{C}$.

Proof. 1. By Lemma 1, we know that $A^{\prime}$ is $G$-graded $\mathcal{O}$-algebra over $C_{A}(B)$ and let $\theta: C_{A}(B) \rightarrow C_{A^{\prime}}\left(B^{\prime}\right)$ to be its G-graded G-acted structure homomorphism. Now, given that $A$ is a strongly G-graded $\mathcal{O}$-algebra over $\mathscr{C}$, we have a G-graded G-acted algebra homomorphism $\zeta: \mathscr{C} \rightarrow \mathrm{C}_{A}(\mathrm{~B})$ and by taking $\zeta^{\prime}: \mathscr{C} \rightarrow \mathrm{C}_{\mathrm{A}^{\prime}}\left(\mathrm{B}^{\prime}\right)$ to be the G-graded G-acted algebra homomorphism obtained by composing $\zeta$ with $\theta$, we obtain that $A^{\prime}$ is also a G-graded $\mathcal{O}$ algebra over $\mathscr{C}$, with its structure given by $\zeta^{\prime}$. Hence, the first statement of this proposition was proved. 
2. Without any loss in generality, we will identify $\mathrm{P}$ with $\mathrm{A} \otimes_{\mathrm{B}} \mathrm{U}$, for some $\mathrm{U} \in \mathrm{B}-$ mod. Following the proof of Lemma 1 we know that $\mathrm{P}$ is a G-graded $\left(A, A^{\prime}\right)$-bimodule. We now check that $\mathrm{P}$ is $\mathrm{G}$-graded $\left(A, A^{\prime}\right)$-bimodule over $\mathscr{C}$. Indeed, we fix $g \in G, p_{g}=a_{g} \otimes u \in P_{g}$ and $c \in \mathscr{C}$. We have:

$$
p_{g} \cdot c=\left(a_{g} \otimes u\right) \cdot c=\left(a_{g} \otimes u\right) \cdot \zeta^{\prime}(c)=a_{g} \zeta(c) \otimes u,
$$

but $a_{g} \in A_{g}$ so there exists a $b \in B$ such that $a_{g}=u_{g} b$, therefore:

$$
\begin{aligned}
p_{g} \cdot c & =u_{g} b \zeta(c) \otimes u=u_{g} \zeta(c) b \otimes u \\
& =u_{g} \zeta(c) u_{g}^{-1} u_{g} b \otimes u=g \zeta(c) a_{g} \otimes u \\
& =\zeta\left({ }^{g} c\right) a_{g} \otimes u=\zeta\left({ }^{g} c\right)\left(a_{g} \otimes u\right) \\
& =\zeta\left({ }^{g} c\right) \cdot p_{g}={ }^{g} c \cdot p_{g} .
\end{aligned}
$$

Henceforth, the last statement of this proposition has been proved.

\section{Graded Morita contexts over $\mathscr{C}$}

We consider the notations given in Section $\S 2$. Let $\mathscr{C}$ be a G-graded G-acted algebra. We introduce the notion of a G-graded Morita context over $\mathscr{C}$, following the treatment given in $[5, \S 12]$.

Definition 4 Consider the following Morita context:

$$
\left(A, A^{\prime}, \tilde{M}, \tilde{M}^{\prime}, f, g\right) \text {. }
$$

We call it a G-graded Morita context over $\mathscr{C}$ if:

1. A and $\mathrm{A}^{\prime}$ are strongly $\mathrm{G}$-graded $\mathcal{O}$-algebras over $\mathscr{C}$;

2. ${ }_{\mathrm{A}} \tilde{M}_{\mathrm{A}^{\prime}}$ and ${ }_{\mathrm{A}^{\prime}} \tilde{\mathrm{M}}_{\mathrm{A}}^{\prime}$ are $\mathrm{G}$-graded bimodules over $\mathscr{C}$;

3. $\mathrm{f}: \tilde{\mathrm{M}} \otimes_{\mathrm{A}^{\prime}} \tilde{\mathrm{M}}^{\prime} \rightarrow \mathrm{A}$ and $\mathrm{g}: \tilde{\mathrm{M}}^{\prime} \otimes_{\mathrm{A}} \tilde{\mathrm{M}} \rightarrow \mathrm{A}^{\prime}$ are G-graded bimodule homomorphisms such that by setting $\mathbf{f}\left(\tilde{\mathfrak{m}} \otimes \tilde{\mathfrak{m}}^{\prime}\right)=\tilde{\mathfrak{m}} \tilde{\mathfrak{m}}^{\prime}$ and $\mathbf{g}\left(\tilde{\mathfrak{m}}^{\prime} \otimes \tilde{\mathfrak{m}}\right)=$ $\tilde{\mathrm{m}}^{\prime} \tilde{\mathrm{m}}$, we have the associative laws:

$$
\left(\tilde{\mathfrak{m}} \tilde{\mathrm{m}}^{\prime}\right) \tilde{\mathfrak{n}}=\tilde{\mathfrak{m}}\left(\tilde{\mathrm{m}}^{\prime} \tilde{\mathfrak{n}}\right) \quad \text { and } \quad\left(\tilde{\mathrm{m}}^{\prime} \tilde{\mathrm{m}}\right) \tilde{\mathfrak{n}}^{\prime}=\tilde{\mathrm{m}}^{\prime}\left(\tilde{\mathrm{m}} \tilde{\mathfrak{n}}^{\prime}\right) \text {, }
$$

for all $\tilde{\mathrm{m}}, \tilde{\mathrm{n}} \in \tilde{M}, \tilde{\mathrm{m}}^{\prime}, \tilde{\mathrm{n}}^{\prime} \in \tilde{M}^{\prime}$.

If $\mathrm{f}$ and $\mathrm{g}$ are isomorphisms, then $\left(\mathrm{A}, \mathrm{A}^{\prime}, \tilde{\mathrm{M}}, \tilde{M}^{\prime}, \mathrm{f}, \mathrm{g}\right)$ is called a surjective G-graded Morita context over $\mathscr{C}$. 
As an example of a G-graded Morita context over $\mathscr{C}$, we have the following proposition which arises from [5, Proposition 12.6].

Proposition 2 Let $\mathrm{A}$ be a strongly $\mathrm{G}$-graded $\mathcal{O}$-algebra over $\mathscr{C}$, let $\mathrm{P}$ be a $\mathrm{G}$ invariant $\mathrm{G}$-graded $\mathrm{A}$-module, let $\mathrm{A}^{\prime}=\operatorname{End}_{\mathrm{A}}(\mathrm{P})^{\text {op }}$ and let $\mathrm{P}^{*}:=\operatorname{Hom}_{\mathrm{A}}(\mathrm{P}, \mathrm{A})$ be the A-dual of $\mathrm{P}$. Then

$$
\left(A, A^{\prime}, P, P^{*},(\cdot, \cdot),[\cdot, \cdot]\right)
$$

is a $\mathrm{G}$-graded Morita context over $\mathscr{C}$, where $(\cdot, \cdot)$ is a $\mathrm{G}$-graded $(\mathrm{A}, \mathrm{A})$-homomorphism, called the evaluation map, defined by:

$$
\begin{aligned}
& (\cdot, \cdot): \mathrm{P} \otimes_{\mathrm{A}^{\prime}} \mathrm{P}^{*} \rightarrow \mathrm{A}, \\
& \chi \otimes \varphi \mapsto \varphi(\mathrm{x}), \text { for all } \varphi \in \mathrm{P}^{*}, x \in \mathrm{P},
\end{aligned}
$$

and where $[\cdot, \cdot]$ is a $\mathrm{G}$-graded $\left(\mathrm{A}^{\prime}, \mathrm{A}^{\prime}\right)$-homomorphism defined by:

$$
\begin{aligned}
& {[\cdot, \cdot]: \mathrm{P}^{*} \otimes_{\mathrm{A}} \mathrm{P} \rightarrow \mathrm{A}^{\prime},} \\
& \varphi \otimes \mathrm{x} \mapsto[\varphi, x], \text { for all } \varphi \in \mathrm{P}^{*}, x \in \mathrm{P},
\end{aligned}
$$

where for every $\varphi \in \mathrm{P}^{*}$ and $\chi \in \mathrm{P},[\varphi, \chi]$ is an element of $\mathrm{A}^{\prime}$ such that

$$
y[\varphi, x]=\varphi(y) \cdot x, \text { for all } y \in P .
$$

Proof. For the sake of simplicity, we will assume that $A$ is a crossed product as in Section $\S 3$. By Proposition 1 , we have that $A^{\prime}$ is also a $G$-graded $\mathcal{O}$-algebra over $\mathscr{C}$ and that $P$ is a $G$-graded $\left(A, A^{\prime}\right)$-bimodule over $\mathscr{C}$. Now, it is known that the $A$-dual of $P, P^{*}:=\operatorname{Hom}_{A}(P, A)$ is a $\left(A^{\prime}, A\right)$-bimodule, where for each $\varphi \in \mathrm{P}^{*}$ and for each $\mathrm{p} \in \mathrm{P}$, we have:

$$
\left(a^{\prime} \varphi a\right)(p)=\left(\varphi\left(p a^{\prime}\right)\right) a,
$$

for all $a^{\prime} \in A^{\prime}$ and $a \in A$. By $[7, \S 1.6 .4$.$] , we know that P^{*}$ is actually a $\mathrm{G}$-graded $\left(A^{\prime}, A\right)$-bimodule, where for all $\mathrm{g} \in \mathrm{G}$, the $\mathrm{g}$-component is defined as follows:

$$
\mathrm{P}_{\mathrm{g}}^{*}=\left\{\varphi \in \mathrm{P}^{*} \mid \varphi\left(\mathrm{P}_{\mathrm{x}}\right) \subseteq \mathrm{A}_{\mathrm{xg}} \text {, for all } x \in \mathrm{G}\right\} .
$$

We prove that $P^{*}$ is a $G$-graded $\left(A^{\prime}, A\right)$-bimodule over $\mathscr{C}$. Consider $g, h \in G$, $\varphi_{\mathrm{g}} \in \mathrm{P}_{\mathrm{g}}^{*}, \mathrm{c} \in \mathrm{C}$ and $\mathrm{p}_{\mathrm{h}} \in \mathrm{P}_{\mathrm{h}}$. We have:

$$
\left(\varphi_{g} c\right)\left(p_{h}\right)=\left(\varphi_{g}\right)\left(p_{h}\right) c .
$$


Because $\left(\varphi_{g}\right)\left(p_{h}\right) \in A_{h g}$ we can choose a homogeneous element $u_{h g} \in A_{h g}$ and $b \in B$ such that $\left(\varphi_{g}\right)\left(p_{h}\right)=u_{h g} b$. Henceforth,

$$
\begin{aligned}
\left(\varphi_{g} c\right)\left(p_{h}\right) & =u_{h g} b c=u_{h g} c b=u_{h g} c u_{h g}^{-1} u_{h g} b={ }^{h g} c u_{h g} b \\
& ={ }^{h g} c\left(\varphi_{g}\right)\left(p_{h}\right)=\left(\varphi_{g}\right)\left({ }^{h g} c p_{h}\right) \\
& =\left(\varphi_{g}\right)\left(p_{h}{ }^{g} c\right)=\left({ }^{g} c \varphi_{g}\right)\left(p_{h}\right)
\end{aligned}
$$

thus $\varphi_{g} c={ }^{g} \mathcal{c} \varphi_{g}$, therefore $P^{*}$ is a G-graded $\left(A^{\prime}, A\right)$-bimodule over $\mathscr{C}$. Next, following $[5, \S 12]$, it is clear that $(\cdot, \cdot)$ and $[\cdot, \cdot]$ are an $(A, A)$-homomorphism and an $\left(A^{\prime}, A^{\prime}\right)$-homomorphism, respectively. We now check if they are graded, as in the sense of $[2, \S 3]$ : Indeed, consider $p_{g} \in P_{g}$ and $\varphi_{h} \in P_{h}^{*}$. We have:

$$
\left(p_{g}, \varphi_{h}\right)=\varphi_{h}\left(p_{g}\right)
$$

which is an element of $A_{g h}$, given the gradation of $P^{*}:=\operatorname{Hom}_{\mathcal{A}}(P, A)$. Also, for every $y_{k} \in P_{k}$, we have:

$$
\left[\varphi_{h}, p_{g}\right]\left(y_{k}\right)=\varphi_{h}\left(y_{k}\right) p_{g},
$$

which is an element of $A_{k h g}$, given the gradation of $\mathrm{P}^{*}$ and of $\mathrm{P}$, therefore $\left[\varphi_{h}, p_{g}\right]$ is an element of $A_{h g}$. Finally, we verify the associative law of the two homomorphism: Let $p, q \in P$ and $\varphi, \psi \in \mathrm{P}^{*}$. We have:

$$
(p, \varphi) q=\varphi(p) q \quad \text { and } \quad p[\varphi, q]=\varphi(p) q
$$

hence

$$
(p, \varphi) q=p[\varphi, q]
$$

and for all $y \in P$, we have:

$$
([\varphi, p] \psi)(y)=\psi(y[\varphi, p])=\psi(\varphi(y) p)=\varphi(y) \psi(p),
$$

because $\varphi(y) \in A$ and $\psi$ is A-linear, and also we have

$$
(\varphi(p, \psi))(y)=(\varphi \psi(p))(y)=\varphi(y) \psi(p),
$$

because $\psi(p) \in A$, hence

$$
[\varphi, p] \psi=\varphi(p, \psi) .
$$

Therefore $\left(A, A^{\prime}, P, P^{*},(\cdot, \cdot),[\cdot, \cdot]\right)$ is a G-graded Morita context over $\mathscr{C}$.

If $\left(A, A^{\prime}, \tilde{M}, \tilde{M}^{\prime}, f, g\right)$ is a surjective G-graded Morita context over $\mathscr{C}$, then by Proposition 12.7 of [5], we have that $A^{\prime}$ is isomorphic to $\operatorname{End}_{\mathcal{A}}(\tilde{M})^{\text {op }}$ and we have a bimodule isomorphism between $\tilde{M}^{\prime}$ and $\tilde{M}^{*}=\operatorname{Hom}_{\mathcal{A}}(\tilde{M}, A)$. Henceforth, in this situation, the example given by Proposition 2 is essentially unique up to an isomorphism.

Given Corollary 12.8 of [5], the example given by Proposition 2 is a surjective G-graded Morita context over $\mathscr{C}$ if and only if ${ }_{A} \mathrm{P}$ is a progenerator. 


\section{$5 \quad$ Graded Morita theorems over $\mathscr{C}$}

Consider the notations given in $\S 2$. Let $\mathscr{C}$ be a G-graded G-acted algebra. We denote by $A$ and $A^{\prime}$ two strongly G-graded $\mathcal{O}$-algebras over $\mathscr{C}$ (with identity components $\mathrm{B}:=\mathrm{A}_{1}$ and $\mathrm{B}^{\prime}:=A_{1}^{\prime}$ ), each endowed with $\mathrm{G}$-graded G-acted algebra homomorphism $\zeta: \mathscr{C} \rightarrow \mathrm{C}_{\mathrm{A}}(\mathrm{B})$ and $\zeta^{\prime}: \mathscr{C} \rightarrow \mathrm{C}_{\mathrm{A}^{\prime}}\left(\mathrm{B}^{\prime}\right)$ respectively. According to [4] we have the following definitions:

Definition 5 1. We say that the functor $\tilde{\mathcal{F}}: \mathrm{A}-\mathrm{Gr} \rightarrow \mathrm{A}^{\prime}$-Gr is $\mathrm{G}$-graded if for every $\mathrm{g} \in \mathrm{G}, \tilde{\mathcal{F}}$ commutes with the $\mathrm{g}$-suspension functor, i.e. $\tilde{\mathcal{F}} \circ \mathrm{T}_{\mathrm{g}}^{\mathcal{A}}$ is naturally isomorphic to $\mathrm{T}_{\mathrm{g}}^{\mathrm{A}^{\prime}} \circ \tilde{\mathcal{F}}$;

2. We say that $\mathrm{A}$ and $\mathrm{A}^{\prime}$ are $\mathrm{G}$-graded Morita equivalent if there is a Ggraded equivalence: $\tilde{\mathcal{F}}: \mathrm{A}-\mathrm{Gr} \rightarrow \mathrm{A}^{\prime}$-Gr.

Assume that $A$ and $A^{\prime}$ are $G$-graded Morita equivalent. Therefore, we can consider the $\mathrm{G}$-graded functors:

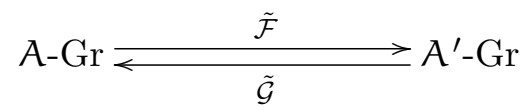

which give a G-graded Morita equivalence between $A$ and $A^{\prime}$. By Gordon and Green's result [4, Corollary 10], this is equivalent to the existence of a Morita equivalence between $A$ and $A^{\prime}$ given by the following functors:

$$
\text { A-Mod } \underset{\mathcal{G}}{\gtrless} A^{\prime}-\operatorname{Mod}
$$

such that the following diagram is commutative:

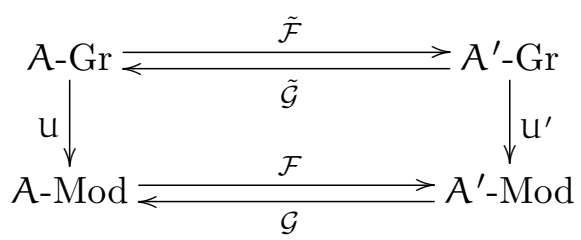

in the sense that:

$$
\mathrm{U}^{\prime} \circ \tilde{\mathrm{F}}=\mathrm{F} \circ \mathrm{U}, \quad \mathrm{U} \circ \tilde{\mathrm{G}}=\mathrm{G} \circ \mathrm{U}^{\prime},
$$

where $\mathrm{U}^{\prime}$ is the forgetful functor from $A^{\prime}$-Gr to $A^{\prime}$-Mod. 
Lemma 2 If $\tilde{\mathrm{P}}$ is a G-graded A-module, then $\tilde{\mathrm{P}}$ and $\tilde{\mathcal{F}}(\tilde{\mathrm{P}})$ have the same stabilizer in $\mathrm{G}$.

Proof. Let $g \in \mathrm{G}_{\tilde{\mathrm{P}}}$. We have $\tilde{\mathrm{P}} \simeq \tilde{\mathrm{P}}(\mathrm{g})$ as G-graded $A$-modules. Because $\tilde{\mathcal{F}}$ is a graded functor, we have that it commutes with the $\mathrm{g}$-suspension functor. Thus $\tilde{\mathcal{F}}(\tilde{\mathrm{P}}(\mathrm{g})) \simeq \tilde{\mathcal{F}}(\tilde{\mathrm{P}})(\mathrm{g})$ in $\mathrm{A}^{\prime}$-Gr. Henceforth, $\tilde{\mathcal{F}}(\tilde{\mathrm{P}}) \simeq \tilde{\mathcal{F}}(\tilde{\mathrm{P}})(\mathrm{g})$ in $A^{\prime}$-Gr, thus $\mathrm{g} \in \mathrm{G}_{\tilde{\mathcal{F}}(\tilde{\mathrm{P}})}$. Hence $\mathrm{G}_{\tilde{\mathrm{P}}} \subseteq \mathrm{G}_{\tilde{\mathcal{F}}(\tilde{\mathrm{P}})}$. The converse, $\mathrm{G}_{\tilde{\mathcal{F}}(\tilde{\mathrm{P}})} \subseteq \mathrm{G}_{\tilde{\mathrm{P}}}$, is straightforward, thus $\mathrm{G}_{\tilde{\mathrm{P}}}=\mathrm{G}_{\tilde{\mathcal{F}}(\tilde{\mathrm{P}})}$.

Consider $\tilde{\mathrm{P}}$ and $\tilde{\mathrm{Q}}$ two G-graded A-modules. We have the following morphism:

$$
\operatorname{Hom}_{A}(\tilde{\mathrm{P}}, \tilde{\mathrm{Q}}) \longrightarrow \operatorname{\mathcal {F}}_{\mathrm{A}^{\prime}}(\tilde{\mathcal{F}}(\tilde{\mathrm{P}}), \tilde{\mathcal{F}}(\tilde{\mathrm{Q}})) .
$$

By following the proofs of Lemma 1 and Proposition 1, we have a G-graded homomorphism from $\mathscr{C}$ to $\operatorname{End}_{\mathcal{A}}(\tilde{\mathrm{P}})^{\text {op }}$ (the composition between the structure homomorphism $\zeta: \mathscr{C} \rightarrow \mathrm{C}_{\mathrm{A}}(\mathrm{B})$ and the morphism $\theta: \mathrm{C}_{\mathcal{A}}(\mathrm{B}) \rightarrow \operatorname{End}_{\mathcal{A}}(\tilde{\mathrm{P}})^{\text {op }}$ from $\left[8\right.$, Lemma 3.2.]) and that $\tilde{P}$ is a $G$-graded $\left(A, \operatorname{End}_{\mathcal{A}}(\tilde{P})^{\text {op }}\right)$-bimodule. Then, by the restriction of scalars we obtain that $\tilde{\mathrm{P}}$ is a right $\mathscr{C}$-module. Analogously $\tilde{Q}, \tilde{\mathcal{F}}(\tilde{\mathrm{P}})$ and $\tilde{\mathcal{F}}(\tilde{\mathrm{Q}})$ are also right $\mathscr{C}$-modules, thus $\operatorname{Hom}_{\mathcal{A}}(\tilde{\mathrm{P}}, \tilde{\mathrm{Q}})$ and $\operatorname{Hom}_{\mathcal{A}}(\tilde{\mathcal{F}}(\tilde{\mathrm{P}}), \tilde{\mathcal{F}}(\tilde{\mathrm{Q}}))$ are G-graded $(\mathscr{C}, \mathscr{C})$-bimodules. This allows us to state the following definition:

Definition 6 1. We say that the functor $\tilde{\mathcal{F}}$ is over $\mathscr{C}$ if the morphism $\tilde{\mathcal{F}}$ $($ see $(*))$ is a morphism of $\mathrm{G}$-graded $(\mathscr{C}, \mathscr{C})$-bimodules;

2. We say that $A$ and $A^{\prime}$ are G-graded Morita equivalent over $\mathscr{C}$ if there is a G-graded equivalence over $\mathscr{C}: \tilde{\mathcal{F}}: A-G r \rightarrow A^{\prime}-\mathrm{Gr}$.

Theorem 1 (Graded Morita I over $\mathscr{C})$ Let $\left(A, A^{\prime}, \tilde{M}, \tilde{M}^{\prime}, f, g\right)$ be a surjective $\mathrm{G}$-graded Morita context over $\mathscr{C}$. Then the functors

$$
\begin{aligned}
& \tilde{M}^{\prime} \otimes_{A}-: A-G r \rightarrow A^{\prime}-\mathrm{Gr} \\
& \tilde{M} \otimes_{A^{\prime}}-: A^{\prime}-\mathrm{Gr} \rightarrow A-\mathrm{Gr}
\end{aligned}
$$

are inverse G-graded equivalences over $\mathscr{C}$.

Proof. Given [2, Theorem 3.2 (Graded Morita I) 6.] we already know that the pair of functors $\tilde{M}^{\prime} \otimes_{A}$ - and $\tilde{M} \otimes_{A^{\prime}}$ - are inverse G-graded equivalences. It remains to prove that they are also over $\mathscr{C}$. We will only prove that the functor $\tilde{M}^{\prime} \otimes_{A}-$ is over $\mathscr{C}$ because the proof for the latter functor is similar. Consider $\tilde{\mathrm{P}}$ and $\tilde{\mathrm{Q}}$ two G-graded A-modules. 
First, we will prove that the morphism

$$
\tilde{M}^{\prime} \otimes_{A}-: \operatorname{Hom}_{A}(\tilde{P}, \tilde{Q}) \rightarrow \operatorname{Hom}_{A^{\prime}}\left(\tilde{M}^{\prime} \otimes_{A} \tilde{P}, \tilde{M}^{\prime} \otimes_{A} \tilde{Q}\right)
$$

is a $(\mathscr{C}, \mathscr{C})$-bimodule homomorphism. Indeed, consider $\varphi \in \operatorname{Hom}_{\mathcal{A}}(\tilde{\mathrm{P}}, \tilde{\mathrm{Q}})$, then $\tilde{M}^{\prime} \otimes_{\mathrm{A}} \varphi \in \operatorname{Hom}_{\mathrm{A}^{\prime}}\left(\tilde{M}^{\prime} \otimes_{\mathrm{A}} \tilde{\mathrm{P}}, \tilde{M}^{\prime} \otimes_{\mathrm{A}} \tilde{Q}\right)$. Consider $\mathrm{c}, \mathrm{c}^{\prime} \in \mathscr{C}$. We only need to prove that

$$
\tilde{M}^{\prime} \otimes_{A}\left(c \varphi c^{\prime}\right)=c\left(\tilde{M}^{\prime} \otimes_{A} \varphi\right) c^{\prime} .
$$

Let $\tilde{\mathrm{m}}^{\prime} \in \tilde{\mathrm{M}}^{\prime}$ and $\tilde{\mathrm{p}} \in \tilde{\mathrm{P}}$. We have:

$$
\left(\tilde{M}^{\prime} \otimes_{A}\left(c \varphi c^{\prime}\right)\right)\left(\tilde{m}^{\prime} \otimes \tilde{p}\right)=\tilde{m}^{\prime} \otimes\left(c \varphi c^{\prime}\right)(\tilde{p})=\tilde{m}^{\prime} \otimes \varphi(\tilde{p} c) c^{\prime}
$$

and

$$
\begin{aligned}
\left(\mathrm{c}\left(\tilde{M}^{\prime} \otimes_{\mathrm{A}} \varphi\right) \mathrm{c}^{\prime}\right)\left(\tilde{\mathrm{m}}^{\prime} \otimes \tilde{\mathrm{p}}\right) & =\left(\left(\tilde{M}^{\prime} \otimes_{\mathrm{A}} \varphi\right)\left(\left(\tilde{\mathrm{m}}^{\prime} \otimes \tilde{\mathrm{p}}\right) \mathrm{c}\right)\right) \mathrm{c}^{\prime} \\
& =\left(\left(\tilde{M}^{\prime} \otimes_{\mathrm{A}} \varphi\right)\left(\tilde{\mathrm{m}}^{\prime} \otimes \tilde{\mathrm{p}} \mathrm{c}\right)\right) \mathrm{c}^{\prime} \\
& =\left(\tilde{\mathrm{m}}^{\prime} \otimes \varphi(\tilde{\mathrm{p}} \mathrm{c})\right) \mathrm{c}^{\prime} \\
& =\tilde{\mathfrak{m}}^{\prime} \otimes \varphi(\tilde{\mathrm{p}} \mathrm{c}) \mathrm{c}^{\prime} .
\end{aligned}
$$

Henceforth $\tilde{M}^{\prime} \otimes_{\mathrm{A}}\left(\mathrm{c} \varphi c^{\prime}\right)=c\left(\tilde{M}^{\prime} \otimes_{\mathrm{A}} \varphi\right) \mathrm{c}^{\prime}$, thus the morphism $\tilde{M}^{\prime} \otimes_{\mathrm{A}}-$ (see $(* *))$ is a $(\mathscr{C}, \mathscr{C})$-bimodule homomorphism.

Second, we will prove that the morphism $\tilde{M}^{\prime} \otimes_{\mathrm{A}}-$ is a G-graded $(\mathscr{C}, \mathscr{C})$ bimodule homomorphism, i.e. it is grade preserving. Consider $g \in G$ and $\varphi_{g} \in \operatorname{Hom}_{\mathcal{A}}(\tilde{P}, \tilde{Q})_{g}$. We want $\tilde{M}^{\prime} \otimes_{A} \varphi_{g} \in \operatorname{Hom}_{\mathcal{A}^{\prime}}\left(\tilde{M}^{\prime} \otimes_{A} \tilde{P}, \tilde{M}^{\prime} \otimes_{A} \tilde{Q}\right)_{g}$, i.e. by $[7, \S 1.2]$, if for some $h \in G$ and $\tilde{m}^{\prime} \otimes \tilde{p} \in\left(\tilde{M}^{\prime} \otimes_{A} \tilde{P}\right)_{h}$, then we must have $\left(\tilde{M}^{\prime} \otimes_{A} \varphi_{g}\right)\left(\tilde{m}^{\prime} \otimes \tilde{p}\right) \in\left(\tilde{M}^{\prime} \otimes_{A} \tilde{Q}\right)_{h g}$. Beforehand, because $\tilde{m}^{\prime} \otimes \tilde{p} \in\left(\tilde{M}^{\prime} \otimes_{A} \tilde{P}\right)_{h}$, by $[7, \S 1.6 .4]$, there exists some $x, y \in G$ with $h=x y$ such that $\tilde{m}^{\prime} \in \tilde{M}_{x}^{\prime}$ and $\tilde{\mathrm{p}} \in \tilde{\mathrm{P}}_{\mathrm{y}}$. We have:

$$
\begin{aligned}
\left(\tilde{M}^{\prime} \otimes_{A} \varphi_{g}\right)\left(\tilde{m}^{\prime} \otimes \tilde{p}\right) & =\tilde{\mathfrak{m}}^{\prime} \otimes_{g_{g}}(\tilde{p}) \in \tilde{M}_{x}^{\prime} \otimes_{A} \varphi_{g}\left(\tilde{\mathrm{P}}_{y}\right) \\
& \subseteq \tilde{M}_{x}^{\prime} \otimes_{A} \tilde{Q}_{y g} \subseteq\left(\tilde{M}^{\prime} \otimes_{A} \tilde{Q}\right)_{x y g} \\
& =\left(\tilde{M}^{\prime} \otimes_{A} \tilde{Q}\right)_{h g} .
\end{aligned}
$$

Henceforth, the morphism $\tilde{M}^{\prime} \otimes_{A}-: \operatorname{Hom}_{A}(\tilde{P}, \tilde{Q}) \rightarrow \operatorname{Hom}_{\mathcal{A}^{\prime}}\left(\tilde{M}^{\prime} \otimes_{A} \tilde{P}, \tilde{M}^{\prime} \otimes_{A}\right.$ $\tilde{\mathrm{Q}})$ is a G-graded $(\mathscr{C}, \mathscr{C})$-bimodule homomorphism.

By Proposition 2 and the observations made in Section $\S 4$, the following corollary is straightforward.

Corollary 1 Let $\mathrm{P}$ be a $\mathrm{G}$-invariant $\mathrm{G}$-graded $\mathrm{A}$-module and $\mathrm{A}^{\prime}=\operatorname{End}_{\mathrm{A}}(\mathrm{P})^{o p}$. If ${ }_{\mathrm{A}} \mathrm{P}$ is a progenerator, then $\mathrm{P} \otimes_{\mathrm{A}^{\prime}}-$ is a $\mathrm{G}$-graded Morita equivalence over $\mathscr{C}$ between $\mathrm{A}^{\prime}-\mathrm{Gr}$ and $\mathrm{A}-\mathrm{Gr}$, with $\mathrm{P}^{*} \otimes_{\mathrm{A}}-$ as its inverse. 
Theorem 2 (Graded Morita II over $\mathscr{C}$ ) Assume that $\mathrm{A}$ and $\mathrm{A}^{\prime}$ are Ggraded Morita equivalent over $\mathscr{C}$ and let

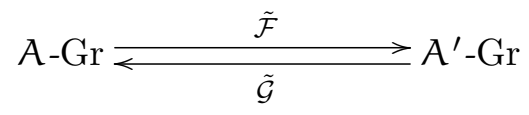

be inverse $\mathrm{G}$-graded equivalences over $\mathscr{C}$. Then this equivalence is given by the following $\mathrm{G}$-graded bimodules over $\mathscr{C}: \mathrm{P}=\tilde{\mathcal{F}}(\mathrm{A})$ and $\mathrm{Q}=\tilde{\mathcal{G}}\left(\mathrm{A}^{\prime}\right)$. More exactly, $\mathrm{P}$ is a $\mathrm{G}$-graded $\left(\mathrm{A}^{\prime}, \mathrm{A}\right)$-bimodule over $\mathscr{C}, \mathrm{Q}$ is a G-graded $\left(\mathrm{A}, \mathrm{A}^{\prime}\right)$-bimodule over $\mathscr{C}$ and the following natural equivalences of functors hold:

$$
\tilde{\mathcal{F}} \simeq \mathrm{P} \otimes_{\mathrm{A}}-\quad \text { and } \quad \tilde{\mathcal{G}} \simeq \mathrm{Q} \otimes_{\mathrm{A}^{\prime}}-
$$

Proof. By $[4$, Corollary 10 (Gordon-Green)], we know that $\mathrm{P}=\tilde{\mathcal{F}}(\mathrm{A})$ is a G-graded $\left(A^{\prime}, A\right)$-bimodule, $Q=\tilde{\mathcal{G}}\left(A^{\prime}\right)$ is a G-graded $\left(A, A^{\prime}\right)$-bimodule and that that the following natural equivalences of functors hold: $\tilde{\mathcal{F}} \simeq \mathrm{P} \otimes_{\mathrm{A}}-$ and $\tilde{\mathcal{G}} \simeq \mathrm{Q} \otimes_{\mathrm{A}^{\prime}}-$. Moreover, we have that ${ }_{\mathrm{A}} \mathrm{P}$ is a progenerator.

It remains to prove that $\mathrm{P}$ and $\mathrm{Q}$ are $\mathrm{G}$-graded bimodules over $\mathscr{C}$. We will only prove that $\mathrm{P}$ is $\mathrm{G}$-graded bimodule over $\mathscr{C}$, because for $\mathrm{Q}$ the reasoning is similar.

By the hypothesis, $A$ and $A^{\prime}$ are G-graded Morita equivalent over $\mathscr{C}$, hence $\tilde{\mathcal{F}}$ and $\tilde{\mathcal{G}}$ are over $\mathscr{C}$. Therefore the function:

$$
\operatorname{Hom}_{A}\left({ }_{A} A,{ }_{A} A\right) \longrightarrow \operatorname{Hom}_{A^{\prime}}\left({ }_{A^{\prime}} P,{ }_{A^{\prime}} P\right)
$$

is an isomorphism of G-graded $(\mathscr{C}, \mathscr{C})$-bimodules, where if $f \in \operatorname{Hom}_{A}\left({ }_{A} A,{ }_{A} A\right)$, we have that $\left(c_{1} f c_{2}\right)(a)=f\left(a c_{1}\right) c_{2}$, for all $a \in A, c_{1}, c_{2} \in \mathscr{C}$. This means that the function

$$
\alpha: A \rightarrow \operatorname{End}_{A^{\prime}}(P)^{\text {op }}, \quad \alpha(a)=\tilde{\mathcal{F}}(\rho(a)), \text { for all } a \in A,
$$

(where $\rho(\mathrm{c}): a \mapsto a c$, for all $a \in A$ ) is an isomorphism of G-graded $(\mathscr{C}, \mathscr{C})$ bimodules. Moreover, by the bimodule structure definition of $\mathrm{P}$ (see [1]), we have that $\alpha(a)(p)=p a$ for all $a \in A$ and for all $p \in P$.

It is clear that ${ }_{A} A$ is $\mathrm{G}$-invariant, hence by Lemma 2, $\mathrm{P}$ is also G-invariant. Henceforth, by Proposition $1, P$ is a G-graded $\left(A^{\prime}, \operatorname{End}_{A^{\prime}}(P)^{\text {op }}\right)$-bimodule over $\mathscr{C}$. Consider the structural homomorphisms $\zeta: \mathscr{C} \rightarrow A, \zeta^{\prime}: \mathscr{C} \rightarrow A^{\prime}$ and $\zeta^{\prime \prime}: \mathscr{C} \rightarrow \operatorname{End}_{A^{\prime}}(\mathrm{P})^{\text {op }}$, thus for all $a \in A$ and for all $c_{1}, c_{2} \in \mathscr{C}$ we have:

$$
\alpha\left(\zeta\left(c_{1}\right) a \zeta\left(c_{2}\right)\right)=\zeta^{\prime \prime}\left(c_{1}\right) \alpha(a) \zeta^{\prime \prime}\left(c_{2}\right) .
$$


By taking $a=1_{\mathrm{A}}$ and $c_{2}=1_{\mathscr{C}}$ we obtain $\alpha \circ \zeta=\zeta^{\prime \prime}$.

Let $g \in \mathrm{G}, \mathrm{p}_{\mathrm{g}} \in \mathrm{P}_{\mathrm{g}}$ and $\mathrm{c} \in \mathscr{C}$. We want $\mathrm{p}_{\mathrm{g}} \cdot \mathrm{c}={ }^{g} \mathrm{c} \cdot \mathrm{p}_{\mathrm{g}}$. We have:

$$
p_{g} \cdot c=p_{g} \cdot \alpha(\zeta(c))=p_{g} \cdot \zeta^{\prime \prime}(c)={ }^{g} \zeta^{\prime}(c) p_{g}={ }^{g} c p_{g} .
$$

Henceforth the statement is proved.

\section{Conclusion}

We have developed a G-graded Morita theory over a G-graded G-acted algebra for the case of finite groups.

In Section §3, we recalled from [9] the notions of a G-graded G-acted algebra, of a G-graded algebra over a G-graded G-acted algebra and that of a G-graded bimodule over a G-graded G-acted algebra and we gave some useful examples for each notion.

In Section $\S 4$, we introduced the notion of a G-graded Morita context over a G-graded G-acted algebra and gave a standard example.

The main results are in Section $\S 5$, where a notion of graded functors over G-graded G-acted algebras and of graded Morita equivalences over G-graded G-acted algebras are introduced and two Morita-type theorems are proved using these notions: We proved that by taking a G-graded bimodule over a G-graded G-acted algebra we obtain a G-graded Morita equivalence over the said G-graded G-acted algebra and that by being given a G-graded Morita equivalence over a G-graded G-acted algebra, we obtain a G-graded bimodule over the said G-graded G-acted algebra, which induces the given G-graded Morita equivalence.

\section{References}

[1] F. W. Anderson, K. R. Fuller, Rings and Categories of Modules, Graduate Texts in Mathematics, Vol. 13, 2nd Ed., Springer-Verlag, BerlinHeidelberg-New York (1992), 55-265.

[2] P. Boisen, Graded Morita Theory, Journal of Algebra, 164 (1994), 1-25.

[3] E. C. Dade, Group-Graded Rings and Modules, Math. Z., 174 (1980), 241-262.

[4] A. del Río, Graded rings and equivalences of categories, Communications in Algebra, 19 (3) (1991), 997-1012. 
[5] C. Faith, Algebra: Rings, Modules and Categories I, Springer-Verlag, Berlin-Heidelberg-New York (1973), 443-453.

[6] R. Gordon, E. L. Green, Graded Artin Algebras, J. of Algebra, 76 (1980), 241-262.

[7] A. Marcus, Representation theory of group-graded algebras, Nova Science (1999), 22-162.

[8] A. Marcus, V. A. Minuță, Group graded endomorphism algebras and Morita equivalences, Mathematica, 62 (85), No 1 (2020), DOI:10.24193/mathcluj.2020.1.08, 73-80.

[9] A. Marcus, V. A. Minuță, Character triples and equivalences over a group graded G-algebra, arXiv:1912.05666, preprint (2019), 1-23.

[10] C. Menini, C. Năstăsescu, When is R-gr Equivalent to the Category of Modules?, J. of Pure and Appl. Algebra, 51 (1988), 277-291.

[11] B. Späth, Reduction theorems for some global-local conjectures, Local Representation Theory and Simple Groups, European Mathematical Society (2018), 23-61.

Received: October 23, 2019 